\title{
Questes
}

\section{Bonnes et mauvaises épouses dans la geste de Guillaume d'Orange : différentes solutions littéraires à la peur de la femme}

\section{Blandine Longhi}

\section{(2) OpenEdition \\ Journals}

Édition électronique

URL : https://journals.openedition.org/questes/2407

DOI : 10.4000/questes.2407

ISSN : 2109-9472

Éditeur

Les Amis de Questes

\section{Édition imprimée}

Date de publication : 15 février 2011

Pagination : 54-66

ISSN : 2102-7188

\section{Référence électronique}

Blandine Longhi, «Bonnes et mauvaises épouses dans la geste de Guillaume d'Orange : différentes solutions littéraires à la peur de la femme », Questes [En ligne], 20 | 2011, mis en ligne le 01 janvier 2014, consulté le 26 août 2021. URL : http://journals.openedition.org/questes/2407 ; DOI : https:// doi.org/10.4000/questes.2407 


\title{
Bonnes et mauvaises épouses dans la geste de Guillaume d'Orange : différentes solutions littéraires à la peur de la femme
}

\author{
Blandine LONGHI
}

La chanson de geste, on le sait, apprécie les oppositions tranchées et ne recule pas devant un certain manichéisme. Les personnages féminins présents dans le cycle de Guillaume d'Orange ne font pas exception à cette tendance. Les deux femmes les plus importantes y sont Guibourc, une épouse modèle soutenant Guillaume en toutes circonstances, et la reine Blanchefleur, présentée comme dépravée, égoïste et de mauvais conseil pour son mari. Bien que les deux personnages ne se croisent pas dans les œuvres, la scène de Laon, lors de laquelle Guillaume répond violemment aux accusations portées par la reine contre Guibourc, permet de cristalliser cette opposition. Autour de ces deux figures gravitent, en arrière-plan, les autres femmes de la famille des Aymerides, qui complètent la palette des représentations de la conjugalité.

Les personnages de Guibourc et de Blanchefleur ont été beaucoup étudiés séparément. J'aimerais m'attacher pour ma part à leur mise en relation, et considérer le fonctionnement qu'ils peuvent avoir en tant que couple antithétique, représentant les deux pôles du comportement féminin possible dans le cadre du mariage. Mon hypothèse est que la femme et la sœur de Guillaume, malgré leurs différences, sont en réalité des réponses littéraires à un même problème : celui de l'angoisse masculine face à la féminité. La fiction construit deux types opposés, qui, par des moyens différents, visent tous deux à conjurer une peur née de la considération de l'altérité féminine. 
Pour étayer cette affirmation, j'aborderai en premier lieu la manière dont se manifeste le rapport entre l'attitude de l'épouse et la virilité de son mari, puis j'analyserai comment les personnages féminins des œuvres correspondent à deux façons antithétiques de rassurer les hommes quant à la dangerosité des femmes.

\section{Influence de l'attitude des femmes sur la virilité des maris}

On peut tout d'abord remarquer que l'opposition majeure entre Guibourc et Blanchefleur réside dans la manière dont elles se comportent avec leurs maris respectifs, et dans la propension qu'elles ont à encourager ou au contraire à brider les qualités chevaleresques de leurs époux.

Guibourc apparaît dans tous les épisodes qui la mettent en scène comme un fidèle appui de son mari, qu'elle encourage dans ses activités chevaleresques. Ce soutien implique tout d'abord qu'elle s'acquitte de ses obligations de maitresse de maison. Elle veille avec attention sur toute la maisnie de Guillaume. Le couple est sans enfant, mais Guibourc apparaît à plusieurs reprises dans une position maternelle vis-à-vis des neveux de son mari, dont elle prend soin avec une grande tendresse. Elle considère de son devoir de les nourrir, de veiller à ce qu'ils ne manquent de rien et de les équiper dignement, afin qu'ils puissent servir leur oncle. L'image d'une Guibourc nourricière est récurrente dans La Chanson de Guillaume: Gui, tout jeune homme participant à sa première bataille, évoque avec nostalgie le soin que sa tante prenait de lui ${ }^{1}$. Dans le même ordre d'idées, on trouve à plusieurs reprises le motif suivant: Guibourc se tenant à côté d'un chevalier de sa famille qui dévore à belles dents les plats qu'elle lui sert. Loin de s'offusquer que les hommes ne lui proposent pas de partager les mets, elle se félicite de les voir manger, considérant leur grand appétit une

\footnotetext{
${ }^{1}$ « Mar vit Guiburc, qui suef me norist, / Qui me soleit faire disner si matin ! / Ore est le terme qu'ele le me soleit offrir ; / Ore ai tel faim, ja me verras morir », La Chanson de Guillaume, François SuARD (éd.), Paris, Livre de poche, 2008, v. 1737-1740.
} 
preuve de valeur, comme dans la scène où elle contemple Girard en train de se rassasier après la bataille de Larchamp :

« Par Deu, bel sire, cist est de vostre lin ;

Qui si mangüe un grant braün porcin,

E a dous traiz beit un cester de vin,

Ben dure guere deit rendre a sun veisin $[\ldots] »^{2}$

L'épouse apparaît ainsi à la place qui doit être la sienne : attentive au bienêtre de son mari et des hommes de la famille, et sachant s'effacer devant les besoins masculins.

Guibourc ne limite pas son aide conjugale au domaine strictement domestique, elle soutient aussi son époux sur le plan militaire. Elle est capable d'anticiper les besoins guerriers de son mari et de préparer le départ au combat en l'absence de celui-ci. Quand Guillaume revient à Orange après sa première bataille à Larchamp, Guibourc a déjà réuni ses barons, pour les tenir prêts à la guerre :

Prist ses messages, ses homes fait mander,

Tant qu'ele en out trente mile de tels :

Les quinze mille furent si apresté

Cum de ferir en bataille champel.

Tuz les demeines en ad Guiburc sevrez,

Sus al paleis les assist al digner,

Chançuns e fables lur fait dire et chanter;

Guiburc meimes les sert de vin aporter. ${ }^{3}$

L'anticipation de son épouse permet au comte de reprendre le combat rapidement. Sans cette initiative, il aurait perdu tout espoir de rattraper les Sarrasins afin de se venger.

Dans certains cas, Guibourc dépasse le stade du simple soutien pratique à son époux et se montre capable de le rappeler à ses devoirs chevaleresques quand il semble les oublier. Lors de la scène sous les murailles d'Orange, c'est elle qui se fait le porte-parole des valeurs

${ }^{2}$ Ibid., v. 1054-1057. La même scène a lieu un peu plus loin avec Guillaume : «E tut mangad les dous gasteals rostiz, / E si que a Guiburc une mie n'en offrid / Ne redresçad la chere ne le vis. / Veist le Guiburc, crollad sun chef, si rist », v. 1416-1419.

${ }^{3}$ Ibid., v. 1232-1239. 
épiques. Comme Guillaume revient vaincu et seul de la bataille, elle fait mine de ne pas le reconnaître sous ses habits de Sarrasin et refuse de lui ouvrir les portes de la ville. Elle argue du fait que jamais son valeureux époux ne s'avouerait aussi vite battu et ne laisserait la victoire si facilement aux païens :

Veit $1 \mathrm{e}^{4}$ Guiburc, comence a plurer :

«Se vus fuissez Willame al curb niés, Ja fust escuse sainte crestiëntez, E cele preie qu'i meinent cels lecchers. $»^{5}$

Face à ces remarques, Guillaume se ressaisit et va mettre en déroute les païens qui passent au loin. Il réfléchit ensuite avec sa femme au moyen de prendre une revanche sur les Sarrasins, malgré les lourdes pertes subies. Guibourc joue ici, selon les termes de Micheline de Combarieu, un « rôle pédagogique $»^{6}$, elle redonne à son époux confiance en ses propres capacités. En lui rappelant ses devoirs de chevalier, elle lui rappelle plus largement les valeurs qui fondent son existence et son identité masculine. Face à un Guillaume momentanément découragé, elle est «la force du héros défaillant $»^{7}$.

Guibourc participe donc pleinement au statut de héros épique de Guillaume. Loin de détourner son époux de ses devoirs chevaleresques, elle s'en porte garante. Cette donnée est inscrite dans le fonctionnement du couple dès sa formation. Dans La Prise d'Orange, Guillaume ne dissocie pas sa volonté de conquérir la ville de sa volonté de séduire Orable, la reine païenne de la cité, la future Guibourc. Projet amoureux et projet chevaleresque sont liés, comme le soulignent les vers jouant sur la

\footnotetext{
${ }^{4}$ Guibourc voit passer au loin une troupe de païens maltraitant des prisonniers français.

${ }^{5}$ Ibid., v. 2267-2269.

${ }^{6}$ Micheline de Combarieu du Grès, L'Idéal humain et l'expérience morale chez les héros des chansons de geste, Publications Université de Provence, diffusion Champion, 1979, p. 441.

${ }^{7}$ Ibid., p. 397.
} 
proximité phonique entre Orange et Orable ${ }^{8}$. De même, après la conversion d'Orable et le mariage, la vie conjugale du couple restera intrinsèquement liée à la vie chevaleresque de Guillaume.

À l'opposé de ce couple se trouve celui que forment Blanchefleur, sœur de Guillaume, et le roi Louis. La reine apparaît comme un personnage égoïste, qui refuse de laisser son mari accomplir son devoir. Dans la scène de Laon, alors que Louis hésite à apporter de l'aide à Guillaume pour défendre Orange contre les païens, Blanchefleur ne rappelle pas à son époux son obligation de suzerain qui consiste à accorder un soutien militaire au comte. Bien plus, lorsque le roi, effrayé par la colère de Guillaume, accepte enfin de venir avec trente mille chevaliers, la reine tente de l'en dissuader :

« Nu ferez, sire ! ço respunt la reïne,

Dame Guiburc fu né en païsmisme

Si set maint art e mainte pute guische.

Ele conuist herbes, ben set temprer mescines,

Tost vus ferreit enherber u oscire.

Willame ert dunc reis et Guiburc reïne,

Si remaindreie doleruse e chaitive. ${ }^{9}$

Blanchefleur ne se soucie ni de l'intérêt de la chrétienté, ni de l'honneur de son mari ; seul son propre sort lui importe. Il semble que ses discours portent d'ailleurs en partie leurs fruits, puisque la suite de l'œuvre nous apprend que Louis, s'il envoie bien quarante mille hommes à son beau-frère, ne se déplace finalement pas en personne. Il argue d'une maladie pour s'en excuser, mais le prétexte ne convainc pas Guibourc qui suppute un mensonge.

Le plus inquiétant concernant le personnage de la reine ne réside en effet pas dans la priorité qu'elle accorde à son seul intérêt, mais dans

\footnotetext{
${ }^{8}$ « Einçois verrai comme Orenge est assise / Et Glorïete, cele tor marberine, / Et Dame Orable, la cortoise roïne. / La seue amor me destraint et jostise ", La Prise d'Orange, Claude RÉGNIER (éd.), Paris, Klincksieck, 1972, v. 356-359.

${ }^{9}$ La Chanson de Guillaume, éd. cit., v. 2590-2596.
} 
l'influence néfaste qu'elle exerce sur les hommes de son entourage, à commencer par son mari. Ses mauvais conseils ne font que renforcer la couardise naturelle de Louis et le conforter dans son comportement antichevaleresque, puisqu'elle proteste dès qu'il envisage d'avoir une attitude digne de sa fonction. Au cercle vertueux qui amène Guillaume et Guibourc à s'encourager mutuellement à agir avec honneur s'oppose ici un cercle vicieux, qui pousse les époux à rivaliser d'égoïsme et de lâcheté. Si l'on observe les insultes que Guillaume adresse à sa sœur dans cette scène, on découvre que, selon lui, la mauvaise influence de Blanchefleur s'étend même à d'autres hommes que son mari et notamment à ses prétendus compagnons de débauche, dont le comte en colère dresse ainsi la liste :

« Tedbald vus fut, le culvert lecchere,

E Esturmi od la malveise chere.

Cil deussent garder Larcham de la gente paene :

Il s'en fuïrent, Vivien remist arere.

Plus de cent prestres vus unt ben coillie,

Forment vus unt cele clume chargee,

Unc n'i volsistes apeler chambrere. $»^{10}$

Il est frappant de constater que ces amants se signalent tous par leur manque de courage et de virilité. Comme le rappelle Guillaume, Thibaut et Estourmi se sont tristement illustrés au début de la chanson en fuyant le champ de bataille et en abandonnant leurs hommes face aux armées païennes. Les «prêtres » ne sont pas non plus réputés pour leur virilité et leur bravoure. Que ces hommes soient ou non réellement les amants de Blanchefleur n'est pas ici la question. Les assertions de Guillaume montrent en tout cas que, dans l'esprit du comte, seuls ceux ayant perdu tout sens de l'honneur fréquentent sa sœur. À moins que ce ne soit elle qui, par son contact néfaste et dangereux, leur fasse perdre leurs qualités viriles?

${ }^{10}$ Ibid., v. 2604-2610. 
Le passage semble à cet égard influencé par les poncifs misogynes diffusés dans les discours cléricaux. Ces derniers insistent sur la dangerosité de la sensualité féminine, cause de la Chute et de bien des déboires pour l'homme. Le penchant pour la luxure est indissociable de la nature féminine, et l'homme doit se méfier des femmes qui l'incitent à pécher et à perdre son âme. Le fait que la sexualité soit au centre des reproches adressés à la reine confirme l'influence de cette vision dans l'élaboration du personnage. Cependant, les travaux de Joël Grisward ont montré que l'archétype d'une femme débauchée n'était pas né au Moyen Âge. Son analyse des récits indo-européens souligne que la mythologie nordique présente elle aussi une reine à la sexualité débridée et tentant de corrompre son mari. Ce personnage incarne selon lui la troisième fonction indo-européenne, qui est liée à l'idée de reproduction, figurée dans ce cas sous la forme négative de la luxure ${ }^{11}$. La permanence du schéma à travers les siècles indique bien qu'il correspond à une représentation permettant d'exprimer des préoccupations fondamentales. Derrière cette image de femme lubrique et dangereuse, c'est l'angoisse masculine face au désir féminin qui se dessine. La psychanalyse a montré que la sexualité féminine était objet de peur pour des hommes qui pouvaient la percevoir comme une puissance dévastatrice, menaçant leur force vitale. L'image du vagin denté par exemple, qui d'ailleurs n'est pas propre à la période médiévale, illustre cette crainte d'un engloutissement et d'un anéantissement de la virilité par le sexe féminin. La sexualité débridée attribuée à Blanchefleur par Guillaume fait donc écho au fait qu'elle pousse son mari à se comporter en

\footnotetext{
${ }^{11}$ Joël Grisward montre que le groupe des Aymerides est structuré à partir de l'idéologie des trois fonctions indo-européennes mises au jour par Georges Dumézil. Les personnages se répartissent en trois groupes : les prêtres, les guerriers et les producteurs. Cette dernière fonction implique l'idée d'abondance féconde, qui peut-être déclinée dans les motifs de richesse, de nourriture ou de sexualité. Cf. Joël H. Grisward, Archéologie de l'épopée médiévale, Paris, Payot, 1981, p. 243 sqq.
} 
lâche ; guidée par sa seule sensualité, la reine tend à détruire la virilité de son mari, qui est ici symbolisée par les devoirs royaux et chevaleresques.

\section{Des réponses antithétiques à l'angoisse masculine}

Les personnages de Guibourc et de Blanchefleur illustrent donc une même problématique : l'influence que la femme peut avoir sur son mari et la façon dont elle peut promouvoir ou amoindrir l'identité virile. Ces deux figures proposent au public deux façons de conjurer l'angoisse née du danger représenté par les femmes.

La reine illustre une vision négative de la féminité, en incarnant de façon hyperbolique les défauts prêtés aux femmes. Dans son cas, le danger est éliminé par la force masculine. La réaction violente de Guillaume réduit à néant la nocivité de Blanchefleur. La critique a relevé l'étrangeté des propos de Guillaume, qui apparaissent comme hors-sujet. Alors que sa sœur accuse Guibourc de ne pas avoir renoncé à la sorcellerie païenne et de vouloir trahir le roi, Guillaume ne répond pas à ces affirmations. Il ne cherche pas à disculper sa femme, mais accuse à la place la reine d'être lubrique, égoïste et débauchée. Dans Aliscans, la violence du propos paraît en outre disproportionnée. Les insultes de Guillaume envers sa sœur y sont toujours aussi vives, mais, à la différence de ce qui se passe dans $L a$ Chanson de Guillaume, elles ne répondent pas à une calomnie de Blanchefleur au sujet de Guibourc ${ }^{12}$. La reine réagit simplement au souhait de Louis de céder tout le royaume à Guillaume :

Blancheflor l'ot, s'est en haut escriee : «Voire, dist ele, s'iere desheritee!

Or ont les deable fete ceste acordee. Mau chief puist prendre par qui est porparlee ! $»^{13}$

12 Pour une analyse comparative de ces deux versions, voir l'article de Jeanne Wathelet-Willem, "Le Roi et la reine dans La Chanson de Guillaume et dans Aliscans ", in Mélanges de littérature du Moyen Âge au XX siècle offerts à Melle Jeanne Lods, Paris, École Normale Supérieure de Jeunes Filles, 1978, p. 558-570.

${ }^{13}$ Aliscans, Claude Régnier (éd.), Paris, Champion, 2007, v. 3185-3188. 
Le comte peut être agacé par l'égoïsme de sa sœur en de telles circonstances, mais son explosion de violence apparaît peu justifiée, d'autant plus qu'il n'a jamais cherché à se faire offrir toute la France. Jacques de Caluwé propose d'interpréter ces propos selon la théorie de René Girard: la reine serait victime d'un «transfert sacrificiel de la violence ». Ne pouvant nier l'origine païenne de Guibourc, le comte adresse à sa sœur des injures gratuites, qui sont par antiphrases autant d'éloges des vertus conjugales de Guibourc ${ }^{14}$. À la luxure reprochée à Blanchefleur correspondrait la vertu de Guibourc ${ }^{15}$, aux goûts luxueux et à l'égoïsme de la reine répondraient la capacité de Guibourc à endurer les privations et son souci constant du bien de son mari, du royaume et de la chrétienté.

En fait, il semble que la cohérence des reproches adressés par Guillaume à sa sœur ne soit pas ce qui importe le plus ici. L'idée qui marque le lecteur est celle d'une grande violence, nécessaire pour ramener la femme, fondamentalement dépravée, à une attitude de soumission qui doit être la sienne. Louis est trop faible pour faire taire sa femme et, de façon générale, pour en canaliser la dangerosité en exerçant sur elle un contrôle strict. Guillaume remplace donc le mari défaillant. Par sa violence

\footnotetext{
${ }^{14}$ Jacques de CALuwÉ, «L'Amour et le mariage, moteurs seconds, dans la littérature épique française et occitane du XII ${ }^{\mathrm{e}}$ siècle ", in Willy VAN HoECKE et Andries Welkenhuysen (dir.), Love and Marriage in the twelfth century, Louvain, Leuven University Press, 1981, p. 180. René Girard estime en effet que toute société cherche à détourner vers une victime "sacrifiable» une violence qui risque de frapper les membres qu'elle entend à tout prix protéger. Blanchefleur tient ici cette fonction de bouc émissaire : en endossant le rôle de la mauvaise femme, elle protège Guibourc, dont l'origine païenne pourrait susciter le rejet. Cf. René Girard, La Violence et le sacré, Paris, Grasset, 1972, p. 63 sqq.

${ }^{15}$ La vertu de Guibourc est un point que les auteurs du cycle soulignent. Jeanne Wathelet-Willem montre ainsi que certains textes tentent de minimiser le fait qu'Orable ait été la femme du roi païen Thibaut avant d'épouser Guillaume. La Prise d'Orange souligne qu'elle est une mal mariée et le narrateur des Enfances Guillaume introduit le motif selon lequel le mariage avec Thibaut n'aurait pas été consommé. Cf. " Guibourc, femme de Guillaume », in Les Chansons de geste du cycle de Guillaume d'Orange, t. III, Les Moniages Guibourc. Hommage à Jean Frappier, Paris, SEDES, 1983, p. 335-356.
} 
verbale et physique (il menace de décapiter sa sour, et va jusqu'à dégainer son épée), il terrorise Blanchefleur qui s'enfuit, humiliée :

Et la roïne s'en fuit eschevelee, Toute marrie, bien resemble desvee.

Dedenz sa chambre s'en est fuiant entree,

De la poor chiet a terre pasmee. ${ }^{16}$

Le personnage se trouve ainsi vidé de son potentiel effrayant, écrasé par la puissance masculine. Mieux encore, la reine reconnaît elle-même son erreur et accepte de se soumettre à l'autorité de son frère :

« Or m'otroit Dex icele destinee

Que vers mon frere puisse estre racordee,

Que ceste chose puisse estre pardonee ${ }^{17}$

Guillaume a donc rétabli l'ordre naturel des choses, en replaçant la femme dans la position qui doit être la sienne : soumise à la domination masculine. Le spectacle de sa colère peut agir comme un exutoire sur le public, qui se sent soulagé de voir la menace de la lubricité féminine céder le pas devant la force virile. Le texte acquiert alors une valeur cathartique, délivrant l'auditoire de la peur que pouvait inspirer la sensualité féminine.

À côté de l'anéantissement de la figure négative incarnée par Blanchefleur, les œuvres proposent des figures positives qui contribuent elles aussi à conjurer l'angoisse de la féminité. Le personnage de Guibourc représente une autre solution littéraire, consistant dans l'effacement de l'altérité. Guibourc ne représente pas un danger puisqu'elle a totalement assimilé les valeurs viriles. Dans son cas, sphères féminines et masculines ne s'opposent plus mais se recouvrent. J'ai déjà dit qu'elle était pour Guillaume une compagne attentionnée, le soutenant dans ses activités chevaleresques. Elle peut aussi, au besoin, prendre part à ces activités normalement dévolues aux hommes. En attendant le retour de Guillaume, elle défend Orange les armes à la main, à la tête des dames de la ville :

${ }^{16}$ Aliscans, éd. cit., v. 3217-3220.

${ }^{17}$ Ibid., v. 3255-3257. 
Dame Guiborc ot la broigne endossee,

L'elme lacié, si ot ceinte l'espee. [...]

Les dames ont maint pierre jetee,

Meint Sarrazin ont la teste quassee,

Qui gisent mort, senglant, gole baee. ${ }^{18}$

Guibourc a revêtu l'équipement caractéristique des chevaliers, symbolisant son intégration à l'univers masculin. Cependant, cette attitude ne devient jamais menaçante pour Guillaume : son épouse ne prétend pas le remplacer ni rivaliser avec lui. Si Guibourc prend les armes, c'est uniquement pour gagner du temps en attendant le retour de son mari. Ce n'est pas elle qui a l'initiative des combats, et elle mène une armée de fortune, une armée de femmes, et non pas les hommes de son mari. Elle seconde au mieux son époux mais sans jamais outrepasser son rôle d'auxiliaire ni menacer la suprématie de Guillaume. Guibourc a parfaitement intégré les valeurs viriles de la chevalerie et ne cherche donc pas à les contester, tout comme elle a, depuis sa conversion, intégré les valeurs de la chrétienté dont elle est devenu l'un des plus ardents soutiens. La représentation de cette femme exceptionnelle a un effet rassurant sur l'auditoire, car elle montre que l'altérité, sentie tout d'abord comme menaçante, peut se transformer en aide précieuse si elle est bien maîtrisée grâce au respect de la hiérarchie entre les époux.

Je souhaiterais, pour terminer, aborder le cas d'une troisième femme, qui vient enrichir la dialectique entre Guibourc et la reine : il s'agit d'Aélis, fille de Blanchefleur, qui épouse Rainouart à la fin d'Aliscans. Ce personnage permet de proposer, d'une façon différente et complémentaire de celui de Guibourc, une voie positive pour conjurer la peur des femmes. Aélis s'oppose en tous points à sa mère. Elle incarne la douceur d'une féminité respectueuse des hommes et attentive à ne pas franchir les limites qui lui sont dévolues. C'est elle qui fait prendre conscience à sa mère de

${ }^{18}$ Ibid., v. 4195-4203. 
l'erreur qu'elle a faite en contredisant Guillaume. C'est également elle qui apaisera la colère de son oncle ; en la voyant si ravissante et si humble, ce dernier pardonnera à sa sœur :

Vint a Guillelme, qui n'i quist compeignie,

Devant lui est la bele agenoillie,

Le pié li a et la jambe embracie. [...]

Guillelmes l'ot, li cuers li atendrie

Por la pucele, qui doucement li prie, [...]. ${ }^{19}$

Tout dans l'attitude d'Aélis indique la soumission : elle s'agenouille, parle doucement, et affirme être prête à subir tous les supplices pour apaiser le courroux de Guillaume ${ }^{20}$. Cette humilité contraste avec la rébellion de Blanchefleur et rachète, aux yeux du comte, la conduite de sa sœur. La peur de la féminité s'évanouit ici dans la représentation d'une femme idéale, ne constituant pas de danger pour l'homme. On pourrait appliquer aux personnages de la mère et de la fille les analyses de Gilbert Durand sur l'imaginaire. Selon lui, l'imaginaire se structure en deux régimes, l'un diurne, l'autre nocturne. Le régime diurne est antithétique : des motifs exprimant la conquête héroïque (symboles ascensionnels, lumineux ou diaïrétiques ${ }^{21}$ ) viennent s'opposer aux motifs angoissants afin de lutter contre la peur ressentie face à ces derniers. Le régime nocturne, au contraire, préfère l'euphémisme et exorcise les craintes plus qu'il ne les combat $^{22}$. Dans le cas de Blanchefleur, la peur représentée par la femme est combattue par la violence de Guillaume qui soumet sa sœur en la menaçant de son épée ; nous sommes dans le régime diurne. Dans le cas d'Aélis, la dangerosité est évacuée du texte au profit d'une idéalisation qui met en

\footnotetext{
${ }^{19}$ Ibid., v. 3324-3342.

${ }^{20}$ « Se il te plest, la teste aie tranchie, / Ou je sois arsse et en un feu lancie », Ibid., v. 3329-3330.

${ }^{21}$ Les symboles diaïrétiques regroupent toutes les images évoquant les armes, comprises au sens concret (glaive, épée) ou figuré (les armes spirituelles que sont par exemple les rituels de purification). Cf. Gilbert Durand, Les Structures anthropologiques de l'Imaginaire, Paris, PUF, 1963, p. 165 sqq.

${ }^{22}$ Ibid., p. 203-204.
} 
avant les qualités attachées à la féminité (beauté, douceur, humilité) et exorcise l'angoisse par le recours au régime nocturne de l'imaginaire. Comme Guibourc, Aélis vient donc faire contrepoint à la menace incarnée par Blanchefleur. Ce jeu d'oppositions des figures féminines permet au texte de présenter une palette de solutions, ayant toutes pour finalité de rassurer l'auditoire masculin quant à la nocivité de l'influence des femmes.

Intégration à la sphère masculine, idéalisation des qualités féminines, ou au contraire violence et humiliation : tous ces traitements littéraires permettent de résoudre l'angoisse masculine d'une dissolution de la virilité dans les liens du mariage. L'effet cathartique des œuvres advient donc de deux façons opposées et complémentaires.

Il est intéressant de souligner en conclusion que ces représentations conjugales se doublent d'une réflexion implicite sur le rapport à l'altérité : les couples vertueux, dans lesquels la femme préserve la virilité du mari, sont des couples qu'on pourrait qualifier, au prix d'un anachronisme, de « mixtes ». Dans le cas de Guibourc et Guillaume, de même que dans celui d'Aélis et Rainouart, l'un des époux est d'origine sarrasine. En revanche le couple négatif incarné par Blanchefleur et Louis appartient exclusivement à l'univers chrétien. Ce fait est sans doute à mettre au compte de l'aspect subversif de la geste de Guillaume, dont Philipp Bennett a souligné la 《 construction carnavalesque $»^{23}$ et qui invite, entre autres, à voir dans l'assimilation des autres cultures une richesse potentielle plutôt qu'une source de menace ${ }^{24}$.

23 Philipp Bennett, «Carnaval héroïque et écriture cyclique dans la geste de Guillaume ", in L'Épopée romane. Actes du XV congrès international Rencesvals, Université de Poitiers, 2002, t. 1, p. 253-264.

${ }^{24}$ À ce sujet, voir Micheline de Combarieu du Grès, «Aliscans ou la victoire des "nouveaux chrétiens" (étude sur Guibourc et Rainouart) ", in Jean Dufournet (dir.), Mourir aux Aliscans, Paris, Champion, 1993, p. 55-77. Elle analyse comment les personnages de convertis apparaissent comme des «figures de la résistance » qui sauvent la chrétienté lorsque les Français en offrent une image faible et divisée. 